\title{
miR-379-5p inhibits proliferation and invasion of the endometrial cancer cells by inhibiting expression of ROR1
}

\author{
Minglin Liang, Hui Chen and Jie Min $\bowtie$ \\ Department of Obstetrics and Gynecology, Union Hospital, Tongji Medical College, Huazhong University of Science and Technology, Wuhan, \\ Hubei Province, 430022, China
}

Endometrial cancer is a common gynecological malignancy, and the incidence of this disease has increased in recent years. Recently, some studies suggested that the expression of miR-379-5p suppressed the metastasis of breast cancer cells. However, whether the expression of miR-379-5p could affect the proliferation, migration and invasion of endometrial cancer is unclear. In this study, we established miR-379-5p overexpression and miR-379$5 p$ inhibition in endometrial cancer cells. Next, EdU and colony formation assays were performed to measure proliferation of endometrial cancer cells. Wound healing and transwell assays were carried out to examine the migration and invasion of these cells. Then, luciferase reporter assay was performed to test the relationship between miR-379-5p and ROR1. Finally, we overexpressed ROR1 in miR-379-5p overexpressing endometrial cancer cells. Colony formation, wound healing and transwell assays were used to measure proliferation, migration and invasion of these cells. The results showed that overexpression of miR-379-5p repressed proliferation, migration and invasion of endometrial cancer cells. Higher levels of miR-379-5p repressed expression of $\mathrm{N}$-cadherin, Vimentin and ZEB1. Overexpression of miR-379-5p also promoted expression of E-cadherin and ZO-1. In addition, miR-379-5p targeted and suppressed expression of ROR1. Overexpression of ROR1 abolished the inhibitory effect of miR-379-5p on proliferation, migration, invasion and EMT of endometrial cancer cells. All of these results indicated that miR-379-5p suppressed proliferation, migration and invasion of endometrial cancer cells by inhibiting the expression of ROR1 and the EMT process.

Keywords: endometrial cancer, miR-379-5p, ROR1, EMT, invasion.

Received: 03 November, 2020; revised: 17 December, 2020; accepted: 07 January, 2021; available on-line: 11 May, 2021

『e-mail: minjie39716@163.com

Abbreviations: ROR1 RTK-like orphan receptor 1; EMT Epithelial mesenchymal transition; miRNA microRNAs

\section{INTRODUCTION}

Endometrial cancer is one of the most common malignant tumors of females (Szarszewska et al., 2019). It is a malignant tumor that occurs in the epithelial tissues of postmenopausal women (Smith et al., 2017). Recently, incidence of endometrial cancer has been increasing around the world. The prognosis for patients with metastatic and recurrent endometrial cancer is often poor (Morice et al., 2016). Therefore, we need to further explore the molecular mechanism of endometrial cancer and develop corresponding targeted drugs to inhibit the occurrence and development of endometrial cancer.
MicroRNAs are small non-coding RNA molecules with 12-25 nucleotides and act as post-transcriptional regulators of target genes through complementary pairing with the sequence of the target genes (Macfarlane \& Murphy, 2010; Liolios et al., 2019). Several studies have suggested that expression of miRNAs was associated with physiological processes including differentiation, apoptosis, senescence and inflammation in the cells (Tutar, 2014; Vishnoi \& Rani, 2017). Furthermore, miRNAs are also associated with the development of multiple types of cancer (Zhang et al., 2014; Chen et al., 2018). MiR-379-5p is one of the miRNAs which could affect the proliferation and metastasis of cancer cells (Cao et al., 2018). Higher levels of miR-379-5p could repress the proliferation and invasion of cervical cancer cells by regulating the levels of targeted proteins (Shi et al., 2018). The expression of miR-379-5p was decreased during the development of breast cancer (Khan et al., 2013). However, the role of miR-379-5p in the development of endometrial cancer is unclear.

The RTK-like orphan receptor 1 (ROR1) is a member of the ROR receptor family. These proteins were associated with the normal development of embryos (Karvonen et al., 2017). ROR1 is the receptor of the non-canonical Wnt/planar cell polarity (PCP) pathway (Janovska et al., 2016). Therefore, expression of ROR1 could also modulate the proliferation, migration and differentiation of cells by regulating the Wnt pathway (Niehrs, 2012). Inhibition of ROR1 restricts the epithelialmesenchymal transition (EMT) and metastasis of triplenegative breast cancer cells (Wang et al., 2018). We found that miR-379-5p has a potential to target ROR1. However, whether the expression of miR-379-5p could regulate the development of endometrial cancer by modulating the expression of ROR1 is unclear. Thus, in this study we aimed to determine the relationship between miR-379-5p and ROR1, and their role in the development of endometrial cancer cells.

\section{MATERIALS AND METHODS}

\section{Cell culture and treatment}

Endometrial cancer cell lines (HEC1B, AN3CA, Ishikawa and RL952) were purchased from ATCC (Manassas, VA, USA). All of these cells were incubated with the 1640 medium (Hyclone, USA). The medium was supplemented with fetal bovine serum (Gibco, USA). Cells were cultured in humidified atmosphere with $5 \% \mathrm{CO}_{2}$. In addition, ROR1 overexpression lentivirus was obtained from Genechem (Shanghai, China). Polybrene (Genechem, Shanghai, 
China) was used to increase transfection efficacy. The mimic and inhibitor of miR-379-5p were designed by Genechem (Shanghai, China).

\section{Cell viability assay (CCK-8)}

Before the experiments, cells were cultured in the 96 well plates $\left(2 \times 10^{3}\right.$ cells per well). Stocks of CCK- 8 were diluted with the culture medium (1:10) and added to the plates after cell adhesion. Then, cells were incubated at $37^{\circ} \mathrm{C}$ for 1 hour. Next, the absorbance of these cells was determined with a spectrophotometer (Thermo Fisher Scientific, USA).

\section{EdU assays}

EdU commercial kit (Beyotime, China) was used for measuring proliferation of endometrial cancer cells, following manufacturer's protocol. The images were photographed with a confocal laser scanning microscope (Olympus, Japan).

\section{Clone formation assays}

HEC1B and AN3CA cells were first resuspended. Then, HEC1B (150 cells per dish) and AN3CA (300 cells per dish) cells were plated in the $60 \mathrm{~mm}$ culture dish. Next, these cells were incubated in an incubator (Thermo Fisher Scientific, USA) for two weeks. Then, the cells were fixed with $70 \%$ ethanol and stained with crystal violet (Thermo Fisher Scientific, USA). Finally, the number of clones was counted under the microscope (Olympus, Japan).

\section{Wound healing assays}

HEC1B and AN3CA cells were resuspended and cultured in $35 \mathrm{~mm}$ culture dishes. After adhesion to the bottom, the cells were cultured with in a serumfree medium for 12 hours. After that, a scratch was created with pipette tips. Next, the scratch was photographed under a microscope (Olympus, Japan). After 24 hours, another photo was taken. At last, width of the scratch was measured under the microscope.

\section{Transwell assays}

Cells in different groups were incubated with a serum-free medium for 12 hours before experiments. Meanwhile, matrix gel (BD, USA) was added into the upper chamber (Corning, USA). The complete medium was added into the lower chamber. Cells on the reverse side of the membrane were observed and stained by crystal violet. Finally, the number of cells was calculated with the imageJ software (National Institutes of Health, USA) after staining.

\section{Luciferase reporter assays}

HEC1B and AN3CA cells were plated into 24 well plates. ROR1 wild type sequence and ROR1 mutant sequence (mutant of 3 '-untranslated region) were established with Genechem according to the instructions. Next, the ROR1 wild type and ROR1 mutant sequences were cloned into plasmids. Then, HEC1B and AN3CA cells were co-transfected with the miR$379-5 p$ mimic or inhibitor and wild type or mutant plasmids. At last, commercial luciferase detection kits (Promega, USA) were used for the detection of fluorescence.

\section{Reverse Transcription-Polymerase Chain Reaction (RT-} PCR)

Total RNA was collected with the RIPA buffer (Thermo Fisher Scientific, USA). Next, reverse transcription was performed to prepare cDNA. The ABI7500 system (Thermo Fisher Scientific, USA) was used for the amplification of cDNA. The results were analyzed with the $2-\Delta \Delta C$ t method. The primers used in this study were miR-379-5p forward primer 5'-GGGGGGTGGTAGACTATGGAA-3' and reverse primer 5'-GTGCGTGTCGTGGAGTCG-3'; U6 forward primer 5'-GCT'TCGGCAGCACATATACTAAAAT-3' and reverse primer 5'-CGCTTCACGAATT'TGCGTGTCAT-3'; ERA forward primer 5'-CCT'TCAAGATAACCACCGAA-3' and reverse primer 5'-GGTCAGGAGTTCGAGACAAG-3'; GAPDH forward primer 5'GAGTCAACGGATT'TGGTCGT-3' and reverse primer 5'-GACAAGCTTCCCGT'TCTCAG-3'.

\section{Western blot}

RIPA buffer (Beyotime, China) was used to extract total proteins. The BCA method was used to measure the concentration of the samples. Next, 10\% SDS PAGE (Beyotime, China) was used for separation of the proteins. After that, the proteins were transferred onto PVDF membranes (Millipore, USA). Primary antibodies were then incubated with the membrane $(1: 1000)$. The primary antibodies used in this study were ROR1 (Abcam, ab91187), ZO-1 (Abcam, ab190085), E-cadherin (Abcam, ab238099), N-cadherin (Abcam, ab76011), Vimentin (Abcam, ab92547), ZEB1 (Abcam, ab203829) and $\beta$-actin (Abcam, ab8226). Next, secondary antibodies (Abcam, ab205718 and ab190475) were incubated with these membranes. Finally, the bands were developed with enhanced chemiluminescence reagents (Thermo Fisher Scientific, USA).

\section{Statistical analysis}

All of the experiments in this study were repeated three times. The data in this study is displayed as the mean \pm S.D. The data was analyzed with the student's $t$ test and made into figures with the Graphpad Prism Software (GraphPad Software Inc., USA). $p<0.05$ was considered as statistically significant.

\section{RESULTS}

\section{MiR-379-5p inhibited proliferation of the endometrial cancer cells}

The expression level of miR-379-5p was determined in endometrial cancer cells (HEC1B, AN3CA, Ishikawa and RL952). The results (Fig. 1A) showed that the levels of miR-379-5p were the lowest in the HEC1B cells and the highest in the AN3CA cells. Therefore, we selected the HEC1B cells to establish miR-379-5p overexpression model and chose the AN3CA cells to establish miR-379$5 p$ inhibition model. Higher levels of estrogen receptor (ER) could promote the metastasis of endometrial cancer cells (Hua et al., 2018). The expression of ERA in AN3CA cells was higher than in the HEC1B cells (Fig. 1B). Next, CCK-8 assays were performed to test the viability of these cells. Cells in the control groups did not receive any treatment. Results (Fig. 1C) showed that the viability of HEC1B cells was decreased after overexpression of miR-379-5p. On the other hand, viability of AN3CA 

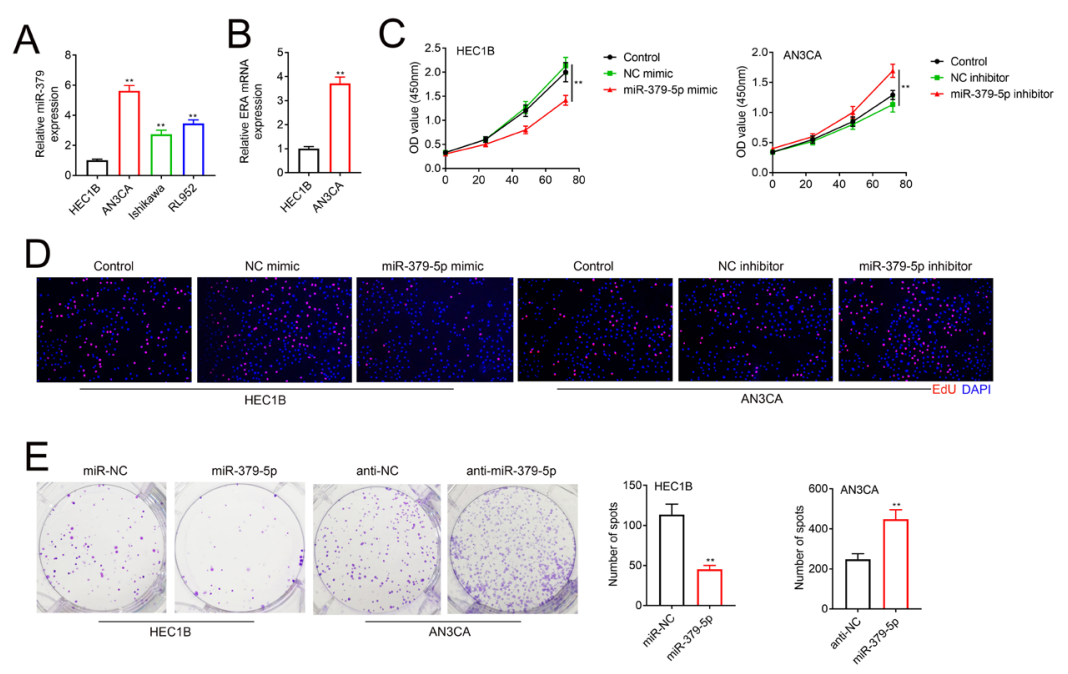

Figure 1. Overexpression of miR-379-5p suppressed proliferation of the endometrial cancer cells.

(A) The expression of miR-379-5p in endometrial cancer cells was measured with RT-PCR. (B) Cell viability of endometrial cancer cells was measured with the CCK-8 assays. (C) Proliferation of endometrial cancer cells was determined with the EdU assays. (D) Proliferation of endometrial cancer cells was measured with colony formation assays. ${ }^{*} p<0.05,{ }^{* *} p<0.01,{ }^{* * *} p<0.001$.

cells was enhanced after inhibition of miR-379-5p. Similarly, results of the EdU assays (Fig. 1D) also revealed that the fluorescence intensity was decreased in the HEC1B cells after overexpression of miR-379-5p and increased in the AN3CA cells after inhibition of miR379-5p. After overexpression of miR-379-5p, the number of colonies (HEC1B) was decreased. At the same time, colony formation was increased after suppression of miR-379-5p in the AN3CA cells (Fig. 1E). These results suggested that miR-379-5p suppressed proliferation of the endometrial cancer cells.

\section{MiR-379-5p suppressed migration and invasion of the endometrial cancer cells}

In this part, we investigated the effect of miR-379$5 p$ on migration and invasion of the endometrial cancer cells. Results of wound healing (Fig. 2A) showed that the ability of scratch healing was decreased in the HEC1B cells after overexpression of miR-379-5p. On the contrary, migration of the AN3CA cells was enhanced after miR-379-5p inhibition. In addition, invasiveness of these cells was determined with transwell assays. According to the results (Fig. 2B), we found that the invasion of

A
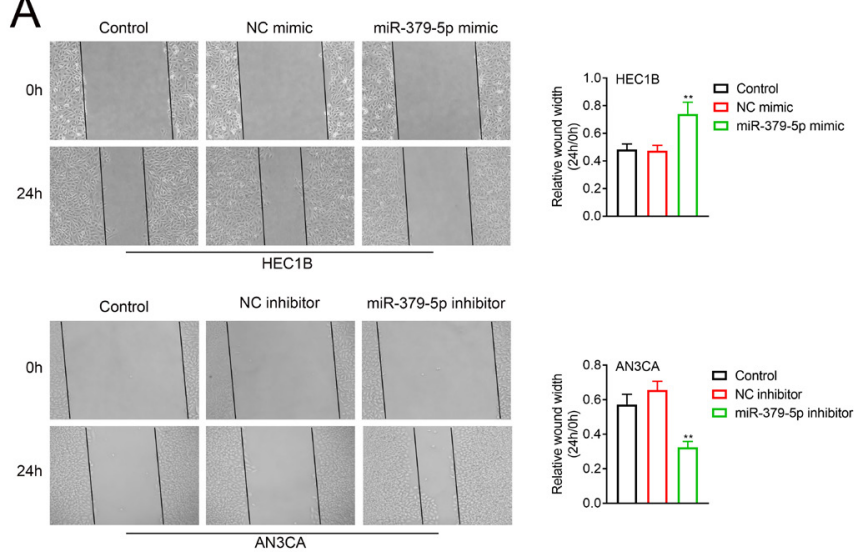

\section{B}
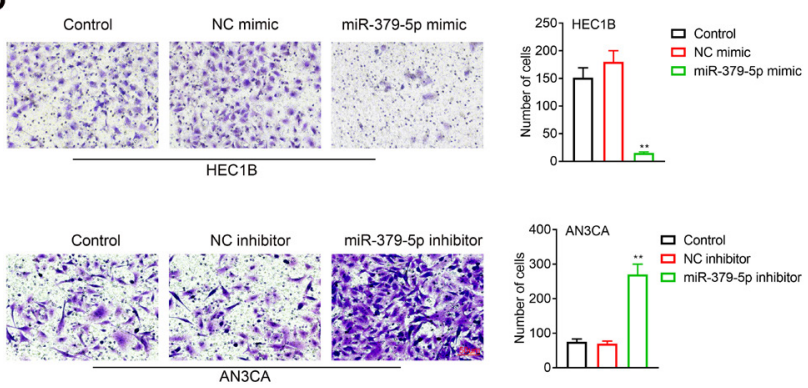

Figure 2. Overexpression of miR-379-5p restricted migration and invasion of the endometrial cancer cells.

(A) The migration of endometrial cancer cells was determined with the wound healing assays. (B) Invasion of endometrial cancer cells was measured with the transwell assays. ${ }^{*} p<0.05,{ }^{* *} p<0.01,{ }^{* * *} p<.001$. 


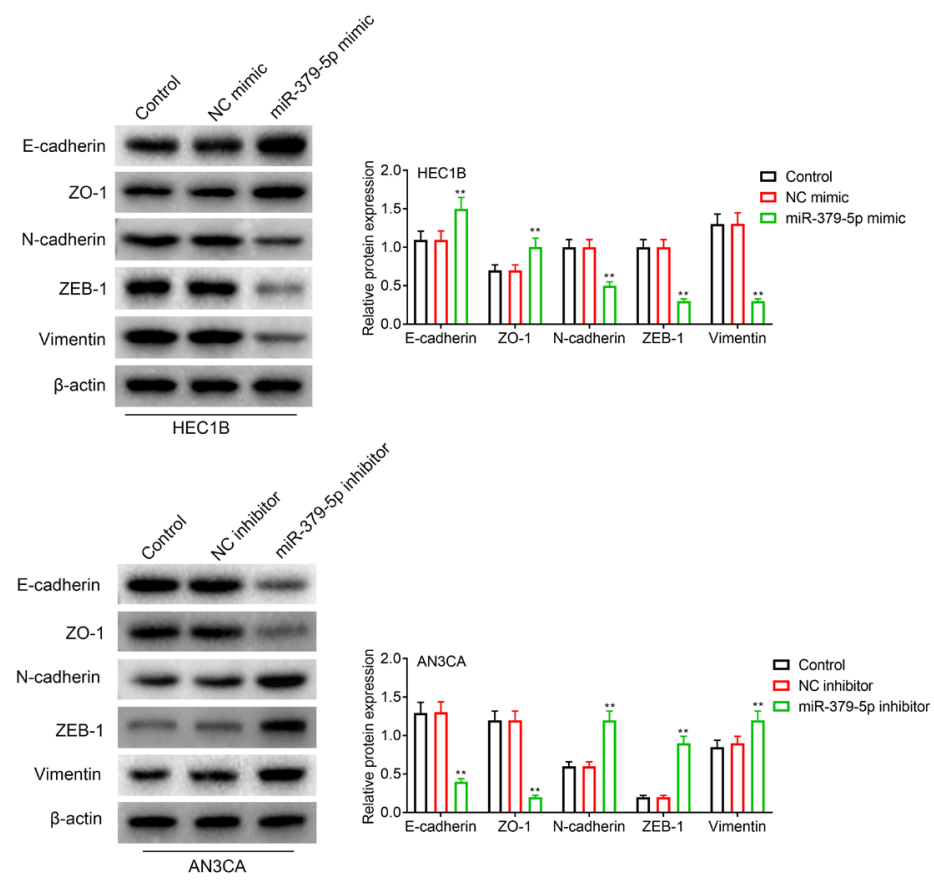

Figure 3. Overexpression of miR-379-5p suppressed the EMT process of the endometrial cancer cells.

The expression of EMT related proteins in endometrial cancer cells was determined by western blot. ${ }^{*} p<0.05,{ }^{* *} p<0.01,{ }^{* * *} p<0.001$.

HEC1B cells was inhibited after overexpression of miR379-5p. Furthermore, inhibition of miR-379-5p promoted invasion of the AN3CA cells. These results revealed that miR-379-5p suppressed migration and invasion of the endometrial cancer cells.

\section{MiR-379-5p restricted development of the Epithelial- Mesenchymal Transition (EMT) process of the endometrial cancer cells}

Proliferation, migration and invasion of multiple types of cancer cells is related to the EMT process. Development of the EMT process could promote metastasis and proliferation of multiple types of cancer cells (Goossens et al., 2017; Suarez-Carmona et al., 2017). Therefore, we explored expression of the EMT related proteins in these cells. As shown in Fig. 3, expression of E-cadherin and ZO-1 was promoted. while the levels of N-cadherin, ZEB1 and Vimentin were decreased in the miR-379-5p overexpressing HEC1B cells. On the other hand, depletion of miR-379-5p in the AN3CA cells had opposite effects. These results implied that miR-379-5p restricted the EMT process in the endometrial cancer cells by suppressing expression of N-cadherin, ZEB1, Vimentin and by promoting expression of E-cadherin and ZO-1.

\section{MiR-379-5p targeted and suppressed expression of ROR1}

Results obtained from the tagertscan database (http:// www.targetscan.org) showed that miR-379-5p has a potential to bind to ROR1 (Fig. 4A). Hence, a luciferase reporter assay was performed to confirm the relationship between miR-379-5p and ROR1. Results (Fig. 4B) revealed that the fluorescence intensity was decreased in the ROR1 wild type and miR-379-5p overexpressing system. Meanwhile, the fluorescence intensity was enhanced in the ROR1 wild type and miR-379-5p inhibition system both, in the HEC1B and AN3CA cells. Next, we determined the levels of ROR1 in the HEC1B and AN3CA cells after overexpression or inhibition of
miR-379-5p. As shown in Fig. 4C, the levels of ROR1 were restricted after overexpression of $\operatorname{miR}-379-5 \mathrm{p}$ in the HEC1B and AN3CA cells. Moreover, expression of ROR1 was promoted after inhibition of miR-379-5p in these cells. Results in this part suggested that miR-379$5 \mathrm{p}$ directly targeted and inhibited expression of ROR1.

\section{Overexpression of ROR1 abolished the inhibitory effect of miR-379-5p on proliferation, migration and invasion of the endometrial cancer cells}

Finally, we overexpressed ROR1 in the miR-379-5p overexpressing HEC1B cells. Results of the CCK-8 assays (Fig. 5A) showed that overexpression of ROR1 rescued viability of the HEC1B cells. Results of the EdU and colony formation assays (Fig. 5B and Fig. 5C) also revealed that overexpression of miR-379-5p suppressed proliferation of the HEC1B cells. However, the fluorescence intensity and the number of colonies were decreased after overexpression of ROR1 in the HEC1B cells. Furthermore, according to the results of the wound healing and transwell assays (Fig. 5D and Fig. 5E), migration and invasion of the HEC1B cells were also rescued after overexpression of ROR1. At last, the expression of EMT related proteins was determined by western blot. The results (Fig. 5F) showed that the expression of E-cadherin and ZO-1 was inhibited in the ROR1 overexpression group. The levels of N-cadherin, ZEB1 and Vimentin were promoted in the HEC1B cells after overexpression of ROR1. All of these results indicated that miR-379-5p inhibited proliferation, migration and invasion of the endometrial cancer cells by inhibiting expression of ROR1.

\section{DISCUSSION}

Endometrial cancer is a crucial cause of death in female cancer patients. In addition, the incidence and mortality of endometrial cancer have been increasing in recent years (Siegel et al., 2018). The endometrial cancer 
A

$$
\begin{array}{rc}
\text { ROR1-WT } & \text { 5'...UAUACCAUAGCAUUUGUCUACCU...3' } \\
\text { hsa-miR-379-5p } & \text { 3' GGAUGCAAGGUAUCAGAUGGU 5' } \\
\text { ROR1-MUT } & \text { 5'...UAUACCAUAGCAUUUCAGAUGGU...3' }
\end{array}
$$

B
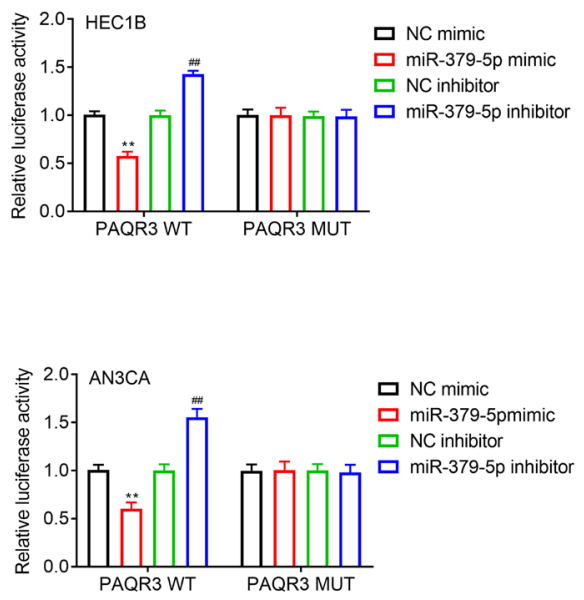
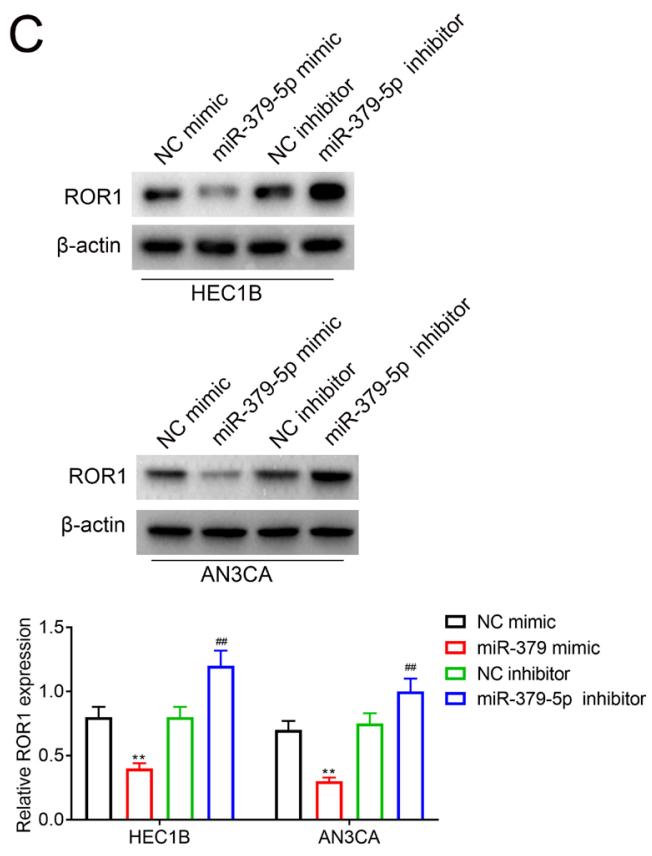

Figure 4. MiR-379-5p targeted and suppressed expression of ROR1 in the endometrial cancer cells.

(A) Potential binding sites between miR-379-5p and ROR1. (B) Fluorescence intensity of these cells was measured with commercial kits. (C) Protein levels of ROR1 in endometrial cancer cells were measured by western blot. ${ }^{*} p<0.05,{ }^{* *} p<0.01,{ }^{* * *} p<0.001$.

A

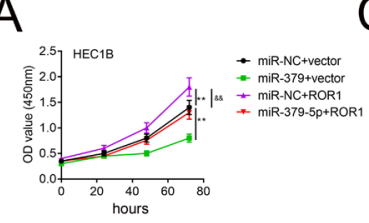

B

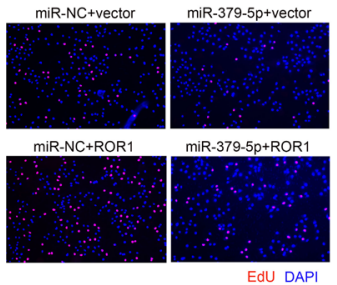

$\mathrm{E}$

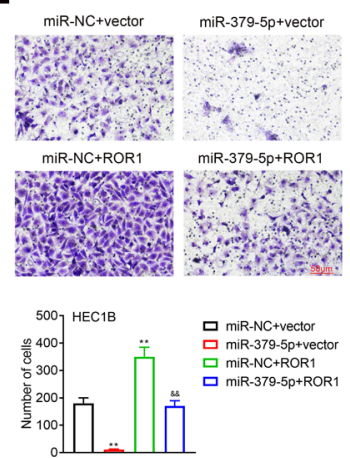

C miR-NC+vector miR-379-5p+vector miR-NC+ROR1
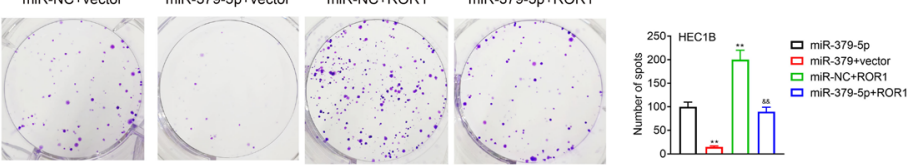

D
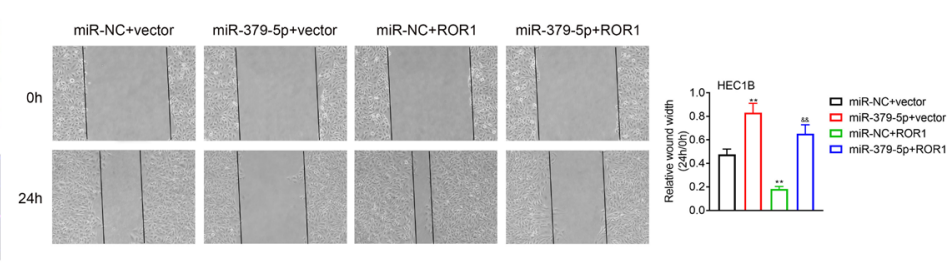

$\mathrm{F}$
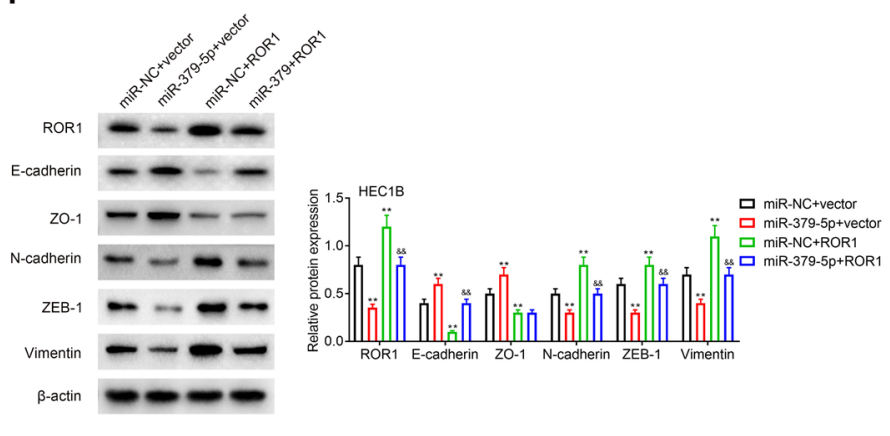

Figure 5. Overexpression of ROR1 suppressed the inhibitory effect of miR-379-5p on proliferation, migration and invasion of the endometrial cancer cells.

(A) CCK-8 was performed to measure the viability of endometrial cancer cells. (B) Proliferation of endometrial cancer cells was measured with the EdU assays. (C) Proliferation of endometrial cancer cells was determined with colony formation assays. (D) Migration of endometrial cancer cells was measured with the wound healing assays. (E) Invasion of endometrial cancer cells was determined with the transwell assays. (F) The expression of EMT related proteins in endometrial cancer cells was measured by western blot. ${ }^{*} p<0.05$, ${ }^{* *} p<0.01$, ${ }^{* * *} p<0.001$. 
cells have shown strong resistance to chemotherapy and radiotherapy. In addition, tumors formed by metastasis of the endometrial cancer cells are more resistant to chemotherapy (Remmerie \& Janssens, 2018). Therefore, it is urgent to explore the mechanism of the occurrence and development of endometrial cancer. In this study, our results revealed the effect of miR-379-5p and ROR1 on proliferation, migration and invasion of the endometrial cancer cells. The conclusion also provided potential strategies for the treatment of the endometrial cancer patients.

MiR-379-5p is a non-coding RNA and plays a crucial role during the development of multiple types of cancer (Nayak et al., 2018; Wei et al., 2019). Some studies also suggested that higher levels of miR-379-5p repressed proliferation and invasion of the cervical cancer cells (Shi et al., 2018), gastric cancer cells (Xu et al., 2017) and oral cancer cells (Shiah et al., 2020) by targeting and regulating expression of the target genes. Moreover, during the development of breast cancer (gynecology malignant tumor), levels of miR-379-5p were decreased in these cells (Khan et al., 2013). In this study, proliferation, migration and invasion of the endometrial cancer cells were enhanced after the inhibition of miR-379-5p. However, proliferation, migration and invasion of these cells were restricted after overexpression of miR-379-5p. These results also implied that the expression of miR$379-5 p$ repressed proliferation, migration and invasion of the endometrial cancer cells.

In addition, miRNAs could affect a physiological process by modulating target gene expression (Wei et al., 2017). Results from a database showed that ROR1 is a potential target gene for miR-379-5p. ROR1 is an evolutionarily conserved receptor protein (Hasan et al., 2019). Higher levels of ROR1 were associated with apoptosis of pancreatic cells (Daneshmanesh et al., 2018), and expression of ROR1 also affected the development of many types of cancer (Balakrishnan et al., 2017). For example, proliferation and invasion of the ovarian cancer cells were inhibited after silencing of ROR1 (Henry et al., 2017). During proliferation and invasion of the endometrial cancer cells, higher levels of ROR1 induced a worse prognosis for patients (Henry et al., 2018). In this study, we revealed that miR-379-5p targeted and repressed expression of ROR1, and the effects of miR-379-5p overexpression on proliferation, migration and invasion of the endometrial cancer cells were suppressed after overexpression of ROR1. These results suggested that miR$379-5 p$ repressed proliferation, migration and invasion of the endometrial cancer cells by suppressing ROR1.

EMT is a physiological process that allows epithelial cells to acquire characteristics of the mesenchymal cells. This process enables many cells, including tumor cells, to acquire strong metastasis and proliferation ability (Ye et al., 2019). In breast cancer cells, the EMT process was restricted after inhibition of ROR1 (Cui et al., 2013). Furthermore, expression of ROR1 also enhanced metastasis of the hepatoma carcinoma cells by promoting the EMT process (Cetin et al., 2019). The ROR1 expression enhanced expression of Vimentin and N-cadherin, and suppressed expression of E-cadherin (Tan et al., 2016). In this study, the results also indicated that the levels of epithelial cell proteins (E-cadherin and ZO-1) were increased after overexpression of miR-379-5p in the endometrial cancer cells. However, the levels of mesenchymal cell proteins (N-cadherin, ZEB1 and Vimentin) were suppressed after overexpression of miR-379-5p in these cells. All of these results indicated that miR-379-5p sup- pressed the endometrial cancer by suppressing expression of ROR1 and development of the EMT process.

\section{CONCLUSION}

Taken together, results of this study revealed that miR-379-5p suppresses proliferation and invasion of the endometrial cancer cells by regulating the expression of ROR1 and the EMT process. However, more research is still needed to carry out animal experiments to further verify this mechanism, and the results of animal experiments will also provide new basis for the development of corresponding targeted drugs.

\section{Acknowledgements}

Not applicable.

\section{Funding}

Not applicable.

\section{Competing interests}

The authors state that there are no conflicts of interest to disclose.

\section{Ethics approval}

Not applicable.

\section{Statement of Human and Animal Rights}

Not applicable.

\section{Statement of Informed Consent}

Not applicable.

\section{Availability of data and materials}

All data generated or analyzed during this study are included in this published article.

\section{Authors' contributions}

Minglin Liang designed the study, supervised data collection, Hui Chen analyzed the data, interpreted the data, Jie Min prepared the manuscript for publication and reviewed the draft of the manuscript. All authors have read and approved the manuscript.

\section{REFERENCES}

Balakrishnan A, Goodpaster T, Randolph-Habecker J, Hoffstrom BG, Jalikis FG, Koch LK, Berger C, Kosasih PL, Rajan A, Sommermeyer D, Porter PL, Riddell SR (2017) Analysis of ROR1 protein expression in human cancer and normal tissues. Clin Cancer Res 23: 3061-3071. https://doi.org/10.1158/1078-0432.Ccr-16-2083

Cao N, Li M, Han J, Wang Y, Wang X (2018) rs61991156 in miR-379 is associated with low capability of glycolysis of gastric cancer by enhanced regulation of PKM2. Cancer Cell Int 18: 92. https://doi. org/10.1186/s12935-018-0593-0

Cetin M, Odabas G, Douglas LR, Duriez PJ, Balcik-Ercin P, YalimCamci I, Sayan AE, Yagci T (2019) ROR1 Expression and its functional significance in hepatocellular carcinoma cells. Cells 8: https:// doi.org/10.3390/cells 8030210

Chen X, Fan Z, McGee W, Chen M, Kong R, Wen P, Xiao T, Chen X, Liu J, Zhu L, Chen R, Wu JY (2018) TDP-43 regulates cancer-associated microRNAs. Protein Cell 9: 848-866. https://doi. org/10.1007/s13238-017-0480-9

Cui B, Zhang S, Chen L, Yu J, Widhopf GF 2nd, Fecteau JF, Rassenti LZ, Kipps TJ (2013) Targeting ROR1 inhibits epithelial-mesenchymal transition and metastasis. Cancer Res 73: 3649-3660. https://doi. org/10.1158/0008-5472.Can-12-3832 
Daneshmanesh AH, Hojjat-Farsangi M, Ghaderi A, Moshfegh A, Hansson L, Schultz J, Vågberg J, Byström S, Olsson E, Olin T, Österborg A, Mellstedt H (2018) A receptor tyrosine kinase ROR1 inhibitor (KAN0439834) induced significant apoptosis of pancreatic cells which was enhanced by erlotinib and ibrutinib. PLoS One 13: e0198038. https://doi.org/10.1371/journal.pone.0198038

Goossens S, Vandamme N, Van Vlierberghe P, Berx G (2017) EMT transcription factors in cancer development re-evaluated: Beyond EMT and MET. Biochim Biophys Acta Rev Cancer 1868: 584-591. https://doi.org/10.1016/j.bbcan.2017.06.006

Hasan MK, Widhopf GF 2nd, Zhang S, Lam SM, Shen Z, Briggs SP, Parker BA, Kipps TJ (2019) Wnt5a induces ROR1 to recruit cortactin to promote breast-cancer migration and metastasis. NPJ Breast Cancer 5: 35. https://doi.org/10.1038/s41523-019-0131-9

Henry C, Hacker N, Ford C (2017) Silencing ROR1 and ROR2 inhibits invasion and adhesion in an organotypic model of ovarian cancer metastasis. Oncotarget 8: 112727-112738. https://doi.org/10.18632/ oncotarget.22559

Henry CE, Llamosas E, Daniels B, Coopes A, Tang K, Ford C (2018) ROR1 and ROR2 play distinct and opposing roles in endometrial cancer. Gynecol Oncol 148: 576-584. https://doi.org/10.1016/j.ygyno.2018.01.025

Hua T, Wang X, Chi S, Liu Y, Feng D, Zhao Y, Wang H (2018) Estrogen-related receptor $\gamma$ promotes the migration and metastasis of endometrial cancer cells by targeting S100A4. Oncol Rep 40: 823832. https://doi.org/10.3892/or.2018.6471

Janovska P, Poppova L, Plevova K, Plesingerova H, Behal M, Kaucka M, Ovesna P, Hlozkova M, Borsky M, Stehlikova O, Brychtova Y, Doubek M, Machalova M, Baskar S, Kozubik A, Pospisilova S, Pavlova S, Bryja V (2016) Autocrine Signaling by Wnt-5a Deregulates Chemotaxis of Leukemic Cells and Predicts Clinical Outcome in Chronic Lymphocytic Leukemia. Clin Cancer Res 22: 459-469. https://doi.org/10.1158/1078-0432.Ccr-15-0154

Karvonen H, Niininen W, Murumägi A, Ungureanu D (2017) Targeting ROR1 identifies new treatment strategies in hematological cancers. Biochem Soc Trans 45: 457-464. https://doi.org/10.1042/ bst20160272

Khan S, Brougham CL, Ryan J, Sahrudin A, O’Neill G, Wall D, Curran C, Newell J, Kerin MJ, Dwyer RM (2013) miR-379 regulates cyclin B1 expression and is decreased in breast cancer. PLoS One 8: e68753. https://doi.org/10.1371/journal.pone.0068753

Liolios T, Kastora SL, Colombo G (2019) MicroRNAs in Female Malignancies. Cancer Inform 18: 1176935119828746. https://doi, org/10.1177/1176935119828746

Macfarlane LA, Murphy PR (2010) MicroRNA: biogenesis, function and role in cancer. Curr Genomics 11: 537-561. https://doi. org/10.2174/138920210793175895

Morice P, Leary A, Creutzberg C, Abu-Rustum N, Darai E (2016) Endometrial cancer. Lancet 387: 1094-1108. https://doi.org/10.1016/ s0140-6736(15)00130-0

Nayak S, Aich M, Kumar A, Sengupta S, Bajad P, Dhapola P, Paul D, Narta K, Purkrait S, Mehani B, Suri A, Chakraborty D, Mukhopadhyay A, Sarkar C (2018) Novel internal regulators and candidate miRNAs within miR-379/miR-656 miRNA cluster can alter cellular phenotype of human glioblastoma. Sci Rep 8: 7673. https://doi. org/10.1038/s41598-018-26000-8

Niehrs C (2012) The complex world of WNT receptor signalling. Nat Rev Mol Cell Biol 13: 767-779. https://doi.org/10.1038/nrm3470

Remmerie M, Janssens V (2018) Targeted therapies in type II endometrial cancers: too little, but not too late. Int J Mol Sci 19: https://doi. org/10.3390/ijms19082380
Shi X, Xiao X, Yuan N, Zhang S, Yuan F, Wang X (2018) MicroRNA-379 suppresses cervical cancer cell proliferation and invasion by directly targeting V-crk avian sarcoma virus CT10 oncogene homolog-like (CRKL). Oncol Res 26: 987-996. https://doi.org/10.3727 /096504017x15140534417184

Shiah SG, Hsiao JR, Chang HJ, Hsu YM, Wu GH, Peng HY, Chou ST, Kuo CC, Chang JY (2020) MiR-30a and miR-379 modulate retinoic acid pathway by targeting DNA methyltransferase $3 \mathrm{~B}$ in oral cancer. J Biomed Sci 27: 46. https://doi.org/10.1186/s12929-02000644-z

Siegel RL, Miller KD, Jemal A (2018) Cancer statistics, 2018. CA Cancer J Clin 68: 7-30. https://doi.org/10.3322/caac.21442

Smith RA, Andrews KS, Brooks D, Fedewa SA, Manassaram-Baptiste D, Saslow D, Brawley OW, Wender RC (2017) Cancer screening in the United States, 2017: A review of current American Cancer Society guidelines and current issues in cancer screening. CA Cancer J Clin 67: 100-121. https://doi.org/10.3322/caac.21392

Suarez-Carmona M, Lesage J, Cataldo D, Gilles C (2017) EMT and inflammation: inseparable actors of cancer progression. Mol Oncol 11: 805-823. https://doi.org/10.1002/1878-0261.12095

Markowska A, Szarszewska M, Żurawski J, Sajdak S, Knapp P, Gryboś A, Olejek A, Bednarek W, Roszak A, Jóźwik M, Marszałek A, Filas V, Wójcik-Krowiranda K, Mądry R, Markowska J, Sozański R (2019) Role of nesfatin in endometrial cancers. Eur J Gynaecolo Oncol 40: 825-830. https://doi.org/10.17219/acem/70861

Tan H, He Q, Gong G, Wang Y, Li J, Wang J, Zhu D, Wu X (2016) miR-382 inhibits migration and invasion by targeting ROR1 through regulating EMT in ovarian cancer. Int J Oncol 48: 181-190. https:// doi.org/10.3892/ijo.2015.3241

Tutar Y (2014) miRNA and cancer; computational and experimental approaches. Curr Pharm Biotechnol 15: 429. https://doi.org/10.2174/1 38920101505140828161335

Vishnoi A, Rani S (2017) MiRNA Biogenesis and regulation of diseases: an overview. Methods Mol Biol 1509: 1-10. https://doi. org/10.1007/978-1-4939-6524-3_1

Wang X, Qiu H, Tang R, Song $\bar{H}$, Pan H, Feng Z, Chen L (2018) miR-30a inhibits epithelial-mesenchymal transition and metastasis in triple-negative breast cancer by targeting ROR1. Oncol Rep 39: 26352643. https://doi.org/10.3892/or.2018.6379

Wei JL, Zhang L, Zhao ZM, Zhao YZ, Fu Q, Yang Y (2019) MicroRNA-379 inhibits laryngeal carcinoma cell proliferation and invasion through directly targeting TCF-4. Kaobsiung J Med Sci 35: 731-738. https://doi.org/10.1002/kjm2.12109

Wei YT, Guo DW, Hou XZ, Jiang DQ (2017) miRNA-223 suppresses FOXO1 and functions as a potential tumor marker in breast cancer. Cell Mol Biol (Noisy-le-grand) 63: 113-118. https://doi.org/10.14715/ $\mathrm{cmb} / 2017.63 .5 .21$

Xu M, Qin S, Cao F, Ding S, Li M (2017) MicroRNA-379 inhibits metastasis and epithelial-mesenchymal transition via targeting FAK/ AKT signaling in gastric cancer. Int J Oncol 51: 867-876. https:// doi.org/10.3892/ijo.2017.4072

Ye F, Tian L, Zhou Q, Feng D (2019) LncRNA FER1L4 induces apoptosis and suppresses EMT and the activation of PI3K/ AKT pathway in osteosarcoma cells via inhibiting miR-18a-5p to promote SOCS5. Gene 721: 144093. https://doi.org/10.1016/j. gene.2019.144093

Zhang Z, Li Z, Li Y, Zang A (2014) MicroRNA and signaling pathways in gastric cancer. Cancer Gene Ther 21: 305-316. https://doi. org/10.1038/cgt.2014.37 